\title{
Analysis of Exponential Backoff with Multipacket Reception in Wireless Networks*
}

\author{
Peng Xuan Zheng, Ying Jun (Angela) Zhang and Soung Chang Liew \\ Department of Information Engineering \\ The Chinese University of Hong Kong \\ \{pxzheng4,yjzhang,soung\}@ie.cuhk.edu.hk
}

\begin{abstract}
A collision resolution scheme is essential to the performance of a random-access wireless network. Most schemes employ exponential backoff (EB) to adjust the transmission attempt rate according to the changing traffic intensity. Previous work on exponential backoff was mostly based on the conventional single-packet-reception model where no more than one packet can be successfully received at any one time. In this paper, we analyze the performance of $E B$ based on a Muti-packet Reception (MPR) model, in which multiple packets can be received successfully at once (i.e., collisions do not occur unless the number of packets transmitted exceeds a threshold that is more than 1). Using a Markov chain model, we derive the throughput expressions for both carrier-sensing and non-carrier-sensing networks with MPR capability under the saturated-traffic condition. We find that the two systems share a number of common performance results. In particular, the state of both systems can be characterized by the same Markov-chain model. The binary exponential backoff (BEB), in which the backoff factor $r$ is set to 2, does not yield the optimum network throughput in both cases. In addition, in both cases, the asymptotic collision probability goes to $1 / r$ and the maximum asymptotic throughput increases roughly linearly with $M$ when the population size approaches infinity. We show how to adjust $r$ to achieve the best throughput performance. Our results show that the optimal $r$ that maximizes the asymptotic throughput increases with $M$ for non-carrier-sensing systems and BEB is close to optimal for carrier-sensing systems. Simulation results validate the accuracy of our theoretical analysis.
\end{abstract}

Index Terms - Multi-packet reception, exponential backoff, wireless networks, ALOHA, WLAN, 802.11, random access.

\section{Introduction}

The collision-resolution scheme used in a random-access wireless network is a significant factor affecting network performance. Core to a collision-resolution scheme is a backoff algorithm which determines how long a node should wait before retransmitting a packet after a packet collision. This is an integral part that determines how well the system adjusts to the ever changing traffic intensity in the network. Exponential backoff (EB), in which each collision causes the

* This work was supported by the Areas of Excellence scheme (Project Number AoE/E-01/99), the Competitive Earmarked Research Grant (Project Number 418506) established under the University Grant Committee of the Hong Kong Special Administrative Region, China, and the Direct Grant (Project Number 2050370) of the Chinese University of Hong Kong. backoff period to be multiplied by a constant factor, has been investigated in detail in the last few decades [1].

Most previous work was based on the traditional singlepacket-reception model. With advanced reception techniques at the physical layer, however, it is possible for the receiver to resolve multiple simultaneously transmitted packets. With Multi-packet Reception (MPR) [2], collisions occur only when the number of simultaneously transmitted packets exceeds the maximum number of simultaneous packets that the receiver can resolve. To date, there has been little work on the behavior and impact of EB under MPR.

In this paper, we analyze the performance of EB with MPR capability under two common random-access models, namely models with and without carrier-sensing. We first derive the throughput expressions for both systems based on a common Markov-chain model assuming a finite population. Then, the asymptotic collision probability and transmission probability for the infinite-population case is studied. We find that the commonly deployed binary exponential backoff (BEB) is not optimal in terms of the overall network throughput. We show how to adjust the backoff factor $r$ to achieve the best performance. In addition, we propose an infinite population model to investigate the asymptotic throughput of EB. Results show that the optimal $r$ which maximizes the asymptotic throughput increases with $M$ for non-carrier-sensing systems and BEB is close to optimal for carrier-sensing systems. Besides, the maximum asymptotic throughput increases roughly linearly with $M$ for both carrier-sensing and noncarrier-sensing systems. The accuracy of our analytical model is validated by extensive simulations.

The rest of the paper is organized as follows. Section 2 introduces the concepts of MPR and EB. Section 3 first describes the analytical model, and then derives the throughput of EB with MPR for the non-carrier-sensing model. Based on the throughput expression, we show how to adjust the backoff factor $r$ for optimal throughput performance. An infinite population model is also presented to analyze the asymptotic behavior of EB when the number of users in the network is large. Section 4 analyzes the performance of an MPR MAC protocol with carrier-sensing. Finally, Section 5 concludes the paper.

\section{Background and Related Work}

\subsection{Mutipacket Reception}

With the advent of CDMA [3] and multiple-antenna [4] techniques, it is no longer a physical constraint for the channel to accommodate only one ongoing transmission. For example, we previously proposed an 802.11-like protocol to support 
MPR in wireless local area networks, where the AP has multiple antennas and is capable of receiving multiple packets simultaneously [5].

Ghez, Verdu and Schwartz [6] were the first to analyze the stability properties of slotted Aloha with MPR capability in the late 1980s. By means of a drift analysis, they showed that the channel backlog Markov chain is ergodic if the packet arrival rate is less than the expected number of packets successfully received in a collision of $n$ packets as $n$ goes to infinity. Not until very recently did MPR resurface in research work again. Researchers from Cornell reopened the topic and have performed extensive studies on various aspects of MPR. In [2], L. Tong et al studied the impact of MPR-enabling signal-processing techniques on the throughput and design of random access protocols. In [7], Zhao and Tong proposed a centralized multiqueue service room MAC protocol (MQSR) which was designed especially for MPR. Later in [8], Zhao and Tong further proposed another centralized MAC protocol for MPR, the dynamic queue protocol, which offers a much simpler implementation and only marginal performance degradation. These medium access schemes all require a central controller to coordinate the transmissions of the client stations. Chan and Berger proposed a cross-layer designed CSMA protocol for MPR (XL-CSMA) in [9]. XL-CSMA is a decentralized random access scheme and may be applied in various situations when a central controller is not available. Work in [5] is the first attempt to implement MPR under the IEEE 802.11 DCF setting in wireless LAN.

In this paper, we assume that the channel has the capability to accommodate up to $M$ simultaneous transmissions. Hereafter, we refer to this as "MPR capability $M$ ". More specifically, this means the packets can be received correctly whenever the number of simultaneous transmissions is no more than $M$. When more than $M$ stations contend for the channel at the same time, no packet can be decoded.

To focus on the effect of MPR on EB, we assume that the channel is error free in the sense that all packet losses are due to collisions (i.e., more than $M$ packets are transmitted at the same time). This assumption is widely used in the literature to simplify analysis and at the same time provide reasonable results. Nonetheless, our work can be easily extended to include the effect of random channel error by adding a term of packet error rate in the throughput expression.

\subsection{Exponential Backoff}

As pointed out in the previous subsection, whenever the number of simultaneous transmissions exceeds the channel's MPR capability, collision happens and the packets involved are garbled. Therefore, once a collision occurs, a collision resolution scheme is needed for the stations to optimally schedule the retransmission of the colliding packets.

One of the most widely used collision resolution protocols is the binary exponential backoff (BEB) scheme, which is being included as part of the MAC specifications in Ethernet [10] and IEEE 802.11 [11] standards. The basic idea of BEB is as follows. In random access networks, a collision implies that the channel is likely to be overloaded. Hence, once a collision occurs, the colliding stations double their contention window size to reduce their transmission probability. In this way, the overall load on the channel is lightened.

Most previous work in this area focuses on the stability issues of EB. Since stability is not the focus of our paper, interested readers are referred to [12] for a more detailed literature survey. In this paper, we assume that EB is "stable" in the sense which will become clear later, and based on that, we perform a careful study of the impact of MPR on the performance of EB.

In this paper, we consider the general form of exponential backoff (EB) in which the backoff factor $r$ can be any value larger than one. The BEB is a special case of the general EB with $r=2$. We also investigate the performance of EB under both carrier-sensing and non-carrier-sensing models. Most of our general conclusions hold for both systems, except that the throughput expressions are different. Although our focus is on the non-carrier-sensing model in Section 3, the Markov-chain model for the analysis of the collision probability and transmission probability also applies to the carrier-sensing case. The difference in the throughputs of the two systems is manifested through the different "time interval" parameters being used for collision event, successful-transmission event, etc. in the throughput expressions.

\section{Analysis of EB with Multipacket Reception}

\subsection{Model}

In the following analysis, we use the so-called time-slotted model, which assumes that the time axis is divided into slots of equal duration. All packet transmissions are of the same length, equal to exactly one slot, and are synchronized. The model assumed here is without carrier-sensing, while the model assumed in Section 4 is with carrier-sensing. Our analysis of the non-carrier-sensing case in this section can be regarded as a generalization of [12] to the MPR framework.

There are $N$ stations, each operating in a saturated mode, which means the stations always have packets waiting for transmission. After each transmission, the transmitting stations will be informed of the outcome of the transmission, i.e., success or failure. We also assume that the feedback time is extremely short compared with the length of packet transmissions, therefore is negligible in our analysis. This assumption is to make sure that our model does not depend on any particular implementation. The throughput expression derived in the next subsection only need to be modified a little bit to account for the effect of any particular feedback mechanism.

The EB algorithm works as follows. Initially, any station having packets to transmit randomly selects a backoff time from 0 to $W_{0}-1$ slots, where the integer $W_{0}$ denotes the minimum contention window size. After waiting for this amount of backoff time, the station transmits a packet in its queue. Every time the transmission is unsuccessful, the contention window for the station will be multiplied by the backoff factor $r$. After $i$ successive failed retransmissions, the number of slots for which the station will wait before the $i+1$ retransmission attempt, $D_{i}$, is generated according to the following distribution [10]: 


$$
\operatorname{Pr}\left\{D_{i}=k\right\}= \begin{cases}\frac{W_{i}+1-F_{i}}{W_{i}\left(W_{i}+1\right)}, & k=0,1, \cdots, W_{i}-1 \\ \frac{F_{i}}{W_{i}+1}, & k=W_{i}\end{cases}
$$

where $W_{i}=\left\lfloor r^{i} W_{0}\right\rfloor$ and $F_{i}=r^{i} W_{0}-W_{i}$. Once a transmission is successful the contention window of that station is reset to $W_{0}$.

We first assume that EB is used in each of the stations in the network, and that with the use of the EB, the stations will finally reach a steady state in which the distribution of the backoff stages of the stations becomes stationary. So we are informally defining the "stability" as the capability of the EB to adapt to the varying traffic condition and finally bring the system into a steady state. We use an infinite-state Markov chain, as in Fig. 1, to model the operation of EB with no retry limit at a station. The reason why no retry limit is introduced is that it is theoretically more interesting to look at the limiting case when $m$ is infinitely large. Besides, by striking out $m$, we are at an advantage of having fewer variables so that clearer relationships between the more interesting parameters can be manifested. Having said that, the analysis in our paper can be easily extended to the case where there is a retry limit $m$. The state in the Markov chain in Fig. 1 is the backoff stage, which is also equal to the number of retransmissions experienced by the station. Therefore, the contention window size is $W_{i}=\left\lfloor r^{i} W_{0}\right\rfloor$ when the station is in state $i$. In our model, we assume that the collision probability of each transmission attempt is equal to a constant $p_{c}$, no matter which state the station is currently at. This assumption is accurate as long as $W_{0}$ and $N$ is large enough [13].

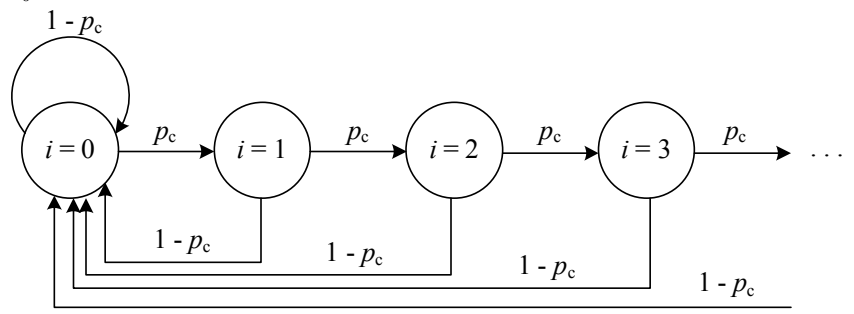

Fig. 1 Markov chain model for the backoff stage.

Let $p_{t}$ be the transmission probability of a station in an arbitrary slot. From this Markov chain, we can derive the expression for $p_{t}$,

$$
p_{t}=\frac{2\left(1-r p_{c}\right)}{W_{0}\left(1-p_{c}\right)+1-r p_{c}} .
$$

where $r p_{c}<1$ is a necessary condition for the steady state to be reachable [12].

In the steady state, the probability that a transmitted packet is collided is equal to the probability that the number of simultaneous transmissions from the other $N-1$ stations in the same slot is no less than the channel's MPR capability. Thus, we have the following relation:

$$
p_{c}=1-\sum_{k=0}^{M-1}\left(\begin{array}{l}
N-1 \\
k
\end{array}\right) p_{t}^{k}\left(1-p_{t}\right)^{N-k-1} .
$$

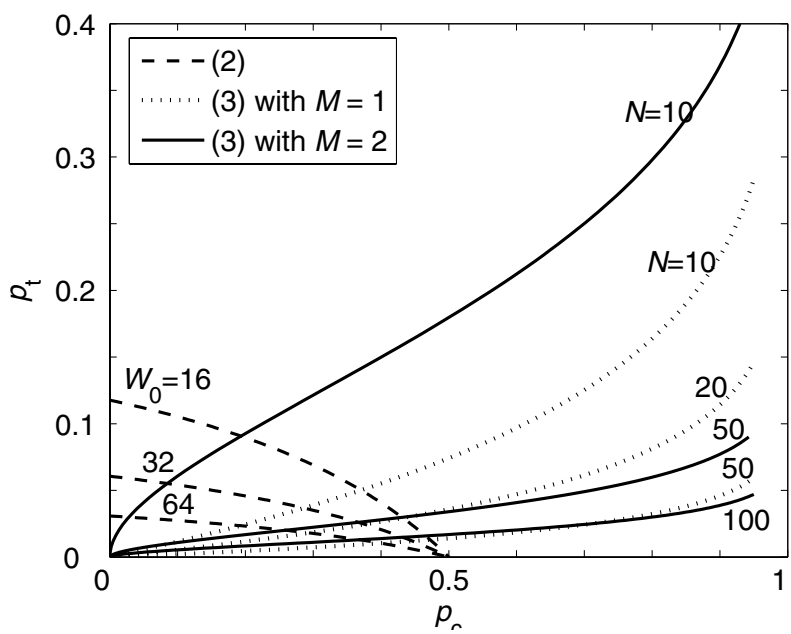

Fig. 2 Plots of $p_{t}$ as a function of $p_{c}$ when $r=2$; dashed lines: $p_{t}$ in (2), dotted lines: $p_{t}$ in (3) with $M=1, r=2$, solid lines: $p_{t}$ in (3) with $M=2$.

From Eqns (2) and (3), we can solve for $p_{c}$ and $p_{t}$, given $N$, $M, r$ and $W_{0}$. The curves determined by (2) and (3) are plotted in Fig. 2. As can be seen, the curves have a unique intersection which can always be calculated numerically. This unique intersection represents the roots $p_{c}$ and $p_{t}$ to Eqns (2) and (3). From the figure, we can also see that as $N$ increases, $p_{t}$ and $p_{c}$ converge to 0 and 0.5 (i.e., $1 / r$ when $r=2$ ) respectively, regardless of $M$. This observation will be proved analytically in the next subsection.

\subsection{Performance Analysis}

Let $P_{t r}$ be the probability that there is at least one transmission in the slot time. Then

$$
P_{t r}=1-\left(1-p_{t}\right)^{N} \text {. }
$$

Let $P_{s k}$ denote the probability that $k$ packets are transmitted simultaneously and the transmission is successful, conditioning on the fact that there is at least one transmission. Since each station transmits its packet independently in a given slot, the probability $P_{s k}$ is calculated as

$$
P_{s k}=\left(\begin{array}{l}
N \\
k
\end{array}\right) p_{t}^{k}\left(1-p_{t}\right)^{N-k} / P_{t r} .
$$
by

Thus, the "normalized throughput" of the network is given

$$
T=\sum_{k=1}^{M} k P_{s k} P_{t r}=\sum_{k=1}^{M} k\left(\begin{array}{l}
N \\
k
\end{array}\right) p_{t}^{k}\left(1-p_{t}\right)^{N-k} .
$$

Note that the throughput can be interpreted as the ratio of the expected number of packets successfully transmitted within one time slot to the maximum number of packets that can be transmitted in that time interval without MPR. Therefore, it has no unit. This definition conforms to the one used in [12] for $M=1$, but slightly different from the throughput definition we use later in the carrier-sensing case.

In the following discussion, we analyze the asymptotic behavior of $p_{c}$ and $T$ in our MPR model. First we show that $p_{c}$ converges to $1 / r$ regardless of the MPR capability $M$.

It is not difficult to see that $\lim _{N \rightarrow \infty} p_{t}=0$ is necessary for the system to reach steady state which is our initial assumption. 
Suppose $p_{t}$ converges to some nonzero value, then $N p_{t}$, the number of average transmission attempts in a slot, approaches infinity as $N$ goes to infinity. This implies that the collision probability $p_{c}=1$, which does not satisfy the necessary condition $p_{c}<1 / r$ for the system to be able to reach the steady state. Hence, $p_{t}$ converges to zero as $N$ goes to infinity.

From (2) we know

$$
p_{t}=\frac{2\left(1-r p_{c}\right)}{W_{0}\left(1-p_{c}\right)+1-r p_{c}} .
$$

Taking the limit, we should have

$$
\lim _{N \rightarrow \infty} p_{t}=\lim _{N \rightarrow \infty} \frac{2\left(1-r p_{c}\right)}{W_{0}\left(1-p_{c}\right)+1-r p_{c}}=0
$$

which implies

Therefore, we have

$$
\lim _{N \rightarrow \infty} 2\left(1-r p_{c}\right)=0
$$

$$
\lim _{N \rightarrow \infty} p_{c}=\frac{1}{r} \quad \text { for all } M .
$$

From (7), we conclude that $p_{c}$ converges to $1 / r$ regardless of $M$ as $N$ goes to infinity. We plot the analytical results of $p_{c}$, obtained by calculating (2) and (3) numerically, as lines and the simulation results as markers in Fig. 3.

To verify the analytical results, we developed a simulator to realize the EB algorithm described in the previous subsection along with those assumptions. The simulator is written in $\mathrm{C}++$ and developed under Visual $\mathrm{C}++6.0$ IDE. The data were collected by running 5,000,000 slots after 1,000,000 slots of warming up, as in [12]. The backoff factor $r$ we use in the simulation is equal to 2, so the general EB reduces to BEB and the minimum contention window sizes we choose are $W_{0}=16,32$, and 64 which conform to the IEEE 802.11 specification for different PHY layers. We only show the simulation results when $M=1$ and 2 as examples and the curves for other $M$ can be inferred from these two special cases.

In Fig. 3, both the curves for $M=1$ and $M=2$ converge to 0.5 (i.e., $1 / r$ since $r=2$ ). We can also see from Fig. 3 that the simulation results match well with the analytical results, which supports our conclusion in (7). It is obvious in the figure that as $N$ increases, $p_{c}$ increases to $1 / r$ more slowly for $M=2$ than for $M=1$. This is self-evident since when the MPR capability is increased it is less likely for a station to collide with others.

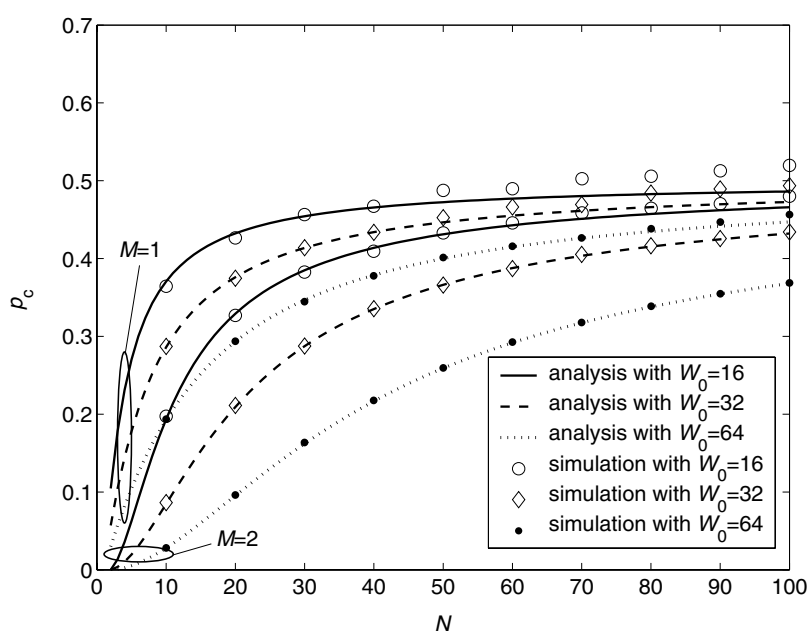

Fig. 3 Plots of $p_{c}$ versus $N$ when $r=2$; lines are analytical results calculated from (2) and (3), markers are simulation results.

We now look at the throughput results based on solving (2), (3) and (6). From Fig. 4, we can see that the throughputs with the same $M$ converge to the same constant as $N$ increases, regardless of $W_{0}$. This phenomenon implies that no matter how crowded the network is, EB can guarantee a nonzero limiting throughput. This limiting throughput only depends on the MPR capability of the channel and is insensitive to the settings of the initial minimum contention window size. In the next subsection, we will show how to predict this "limiting throughput" from the infinite-population model. As shown in Fig. 4, however, this limiting throughput increases as $M$ gets larger. This fact conforms to our intuition, since we would expect higher throughput with increased MPR capability.

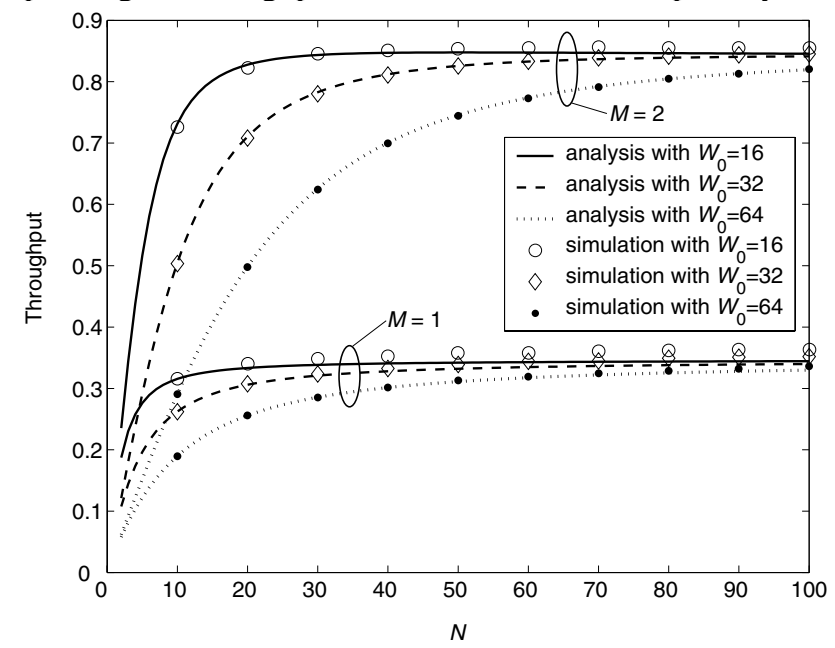

Fig. 4 Plots of throughput versus $N$ when $r=2$; lines are analytical results obtained numerically, markers are simulation results.

As a special case of our general MPR model when $M=1$, Kwak et al [12] proved that, under the traditional collision model, the collision probability $p_{c}$ and network throughput $T$ converge as the number of stations $N$ goes to infinity as follows:

$$
\lim _{N \rightarrow \infty} p_{c}=\frac{1}{r}
$$




$$
\lim _{N \rightarrow \infty} T=\frac{r-1}{r} \ln \frac{r}{r-1} .
$$

Another dimension to investigate the throughput is to see how the throughput behaves as $M$ increases given a fixed number of stations $N$. The analytical results obtained when $N=50$ are plotted in Fig. 5. In this figure, the three curves go up to their maximum value as $M$ increases. The maximum throughput decreases as $W_{0}$ increases. The reason is as follows. First, it is obvious that the maximum throughput is reached when $M=N$. In this case, there is no collision and all transmissions are successful. Therefore, the contention window size is always equal to $W_{0}$. It can be easily figured out that the maximum throughput is equal to $2 N /\left(W_{0}+1\right)$. This implies that the maximum throughput decreases monotonically with $W_{0}$ when $N$ is fixed. Another observation is that, when $M$ is much smaller than $N$, throughput increases roughly linearly with $M$; when $M$ approaches $N$, the throughput reaches the maximum. We will show in the next subsection that the asymptotic throughput (throughput when $N \rightarrow \infty$ ) increases almost linearly with $M$ irrespective of $W_{0}$.

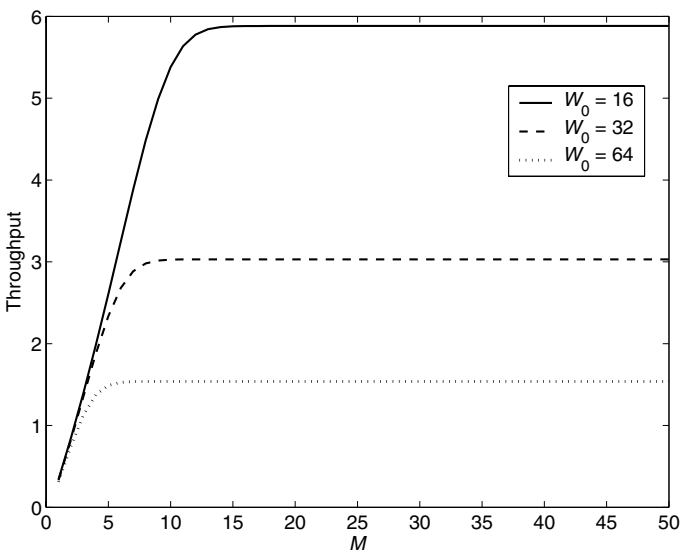

Fig. 5 Throughput versus $M$ when $r=2$ and $N=50$.

In practice, MPR capability is achieved by increasing both the hardware and computational costs. It is therefore worthwhile investigating the achievable throughput per unit cost in our system. As shown in Fig. 5, the throughputs become almost flat as $M$ becomes larger than some value. Therefore, the normalized throughput (normalized by $M$ ) actually increases as $M$ increases at first and then goes down. The optimal point should be somewhere around the corner of the curve. While this is true for finite $N$, for infinite $N$ the normalized throughput increases monotonically with $M$.

Similarly, the relation between throughput and $r$ given $M$ can also be obtained from (2), (3) and (6). Fig. 6 shows the throughput variation with different settings of $r$. From this figure, we can see that, given $M$, there exists an optimal value of $r$ which maximizes the network throughput. This fact can be interpreted as follows. When we increase $r$, the collision probability is reduced. However, we may tend to backoff too much, thus also reducing the transmission probability. Thus, to maximize the throughput by calibrating the backoff factor $r$, we are indeed balancing between letting the stations be more aggressive at the risk of higher collision probability and letting them be conservative at the risk of wasting precious air time when nobody transmits at all. Mathematically, the optimal value of $r$ which maximizes the throughput can be obtained by solving the equation

$$
d T / d r=0 \text {. }
$$

As shown in (9), when $M=1$, the asymptotic throughput is expressed as a function of $r$. The optimal $r$ which maximizes the asymptotic throughput is given by [12]

$$
r_{\text {opt }}=1 /\left(1-e^{-1}\right) \text {. }
$$

However, when $M$ is larger than 1, it is difficult to express $T$ as a closed-form function of $r$, so numerical methods are needed to find the optimal $r$. Another observation from this figure is that the commonly deployed binary exponential backoff is not optimal in the sense that $r=2$ does not achieve the maximum aggregate throughput.

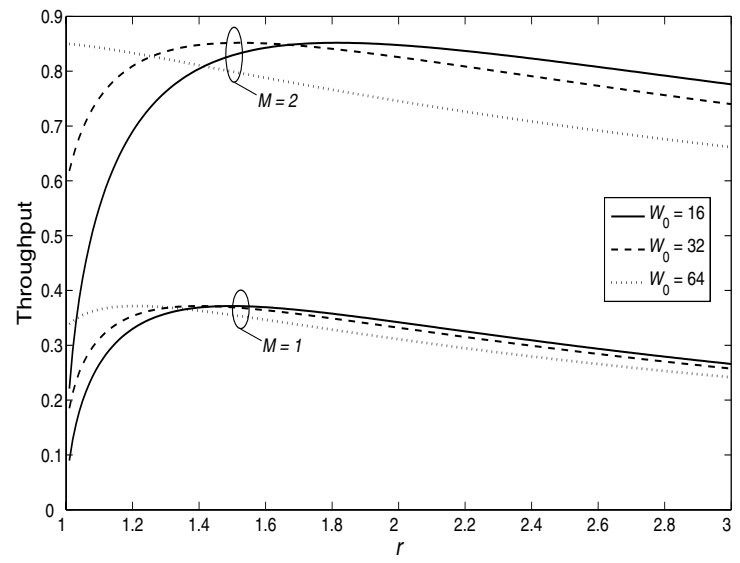

Fig. 6 Throughput versus backoff factor $r$ when $N=50$.

\subsection{Infinite Population Model}

In the previous subsection, we studied EB and achievable throughput of wireless networks with MPR when the number of stations is finite and equal to $N$. The asymptotic performance when $N$ approaches infinity can be obtained by setting $N$ to be a very large number in our previously derived equations. However, this would make the numerical results difficult to obtain. In this subsection, we therefore adopt an alternative infinite-population model to analyze the asymptotic performance of MPR. In this model, it is assumed that the number of stations $N$ in the network is infinitely large and each station independently transmits in a slot with probability $p_{t}$. Recall that $\lim _{N \rightarrow \infty} p_{t}=0$, while $\lim _{N \rightarrow \infty} N p_{t}$ is a constant. Therefore, the originally binomially distributed number of transmission attempts in a slot can be approximated by Poisson distribution:

$$
\operatorname{Pr}\{X=n\}=\frac{\lambda^{n}}{n !} e^{-\lambda}
$$

where the random variable $X$ denotes the number of attempts in a slot and $\lambda$ is the mean which can be expressed as

$$
\lambda=\lim _{N \rightarrow \infty} N p_{t} .
$$

In (7), we have shown that

$$
\lim _{N \rightarrow \infty} p_{c}=\frac{1}{r} \text {. }
$$

Meanwhile, from our infinite population model, we have 


$$
\lim _{N \rightarrow \infty} p_{c}=\operatorname{Pr}\{X \geq M\}=1-\operatorname{Pr}\{X \leq M-1\} .
$$

Thus from (7) and (14), we get the following equation

$$
\operatorname{Pr}\{X \leq M-1\}=\sum_{k=0}^{M-1} \frac{\lambda^{k}}{k !} e^{-\lambda}=e^{-\lambda} \sum_{k=0}^{M-1} \frac{\lambda^{k}}{k !}=1-\frac{1}{r}
$$

which relates $\lambda$ with the values of $M$ and $r$. Hence, we can always calculate $\lambda$ numerically from (15) given $M$ and $r$.

Finally the asymptotic throughput is given by

$$
\begin{aligned}
\lim _{N \rightarrow \infty} T & =\sum_{k=1}^{M} k \operatorname{Pr}\{X=k\}=\lambda \sum_{k=0}^{M-1} \operatorname{Pr}\{X=k\} \\
& =\lambda \operatorname{Pr}\{X \leq M-1\}=\lambda\left(1-\frac{1}{r}\right)
\end{aligned} .
$$

Note that the asymptotic throughput is expressed by $\lambda$ and $r$. Since $\lambda$ is determined by $M$ and $r$, the asymptotic throughput is indeed only a function of $M$ and $r$.

Given $r$ and $M$, we can calculate $\lambda$ from (15). The asymptotic throughput is then obtained by plugging the value of $\lambda$ into (16). Fig. 7 is a plot of the asymptotic throughput versus $r$ for various $M$. From this figure, we can see that the optimal $r$ which maximizes the asymptotic throughput increases with $M$. This result sounds counterintuitive, because we usually expect that with larger $M$ we should decrease $r$ to encourage the stations to be more aggressive. It is true that increasing $r$ will reduce the number of attempts in a slot, but on the other hand this will increase the success probability of an attempt. It is the weighting of these two effects that decides the optimal operating point of $r$. From the results, we can conclude that as $M$ increases, the second effect dominates and in the end moves $r$ to the right to achieve the maximum asymptotic throughput. Another direct conclusion from this figure is that BEB $(r=2)$ is far from optimal. For example, when $M=10 \mathrm{BEB}$ only achieves about 80 percent of the maximum asymptotic throughput.

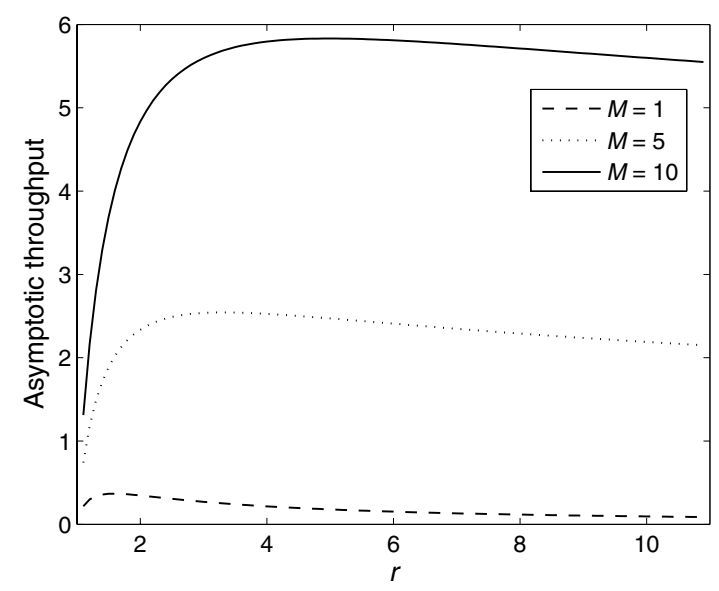

Fig. 7 Asymptotic throughput versus $r$

By tuning $r$ to the optimal value for each $M$, we plot the maximum asymptotic throughput against $M$ in Fig. 8. Unlike Fig. 5 in which $N$ is finite, Fig. 8 shows that the asymptotic throughput increases almost linearly with $M$. In fact, the results are consistent with our observations from Fig. 5 in the sense that throughput increases roughly linearly with $M$ when $N$ (infinitely large here) is much larger than $M$. A close observation of Fig. 8 indicates that the slope of the curve increases slowly with $M$. Consequently, throughput normalized by $M$ also increases with $M$. This implies that as $M$ increases, the achievable throughput per unit cost (i.e., bandwidth in CDMA systems or antenna in multi-antenna systems) also increases.

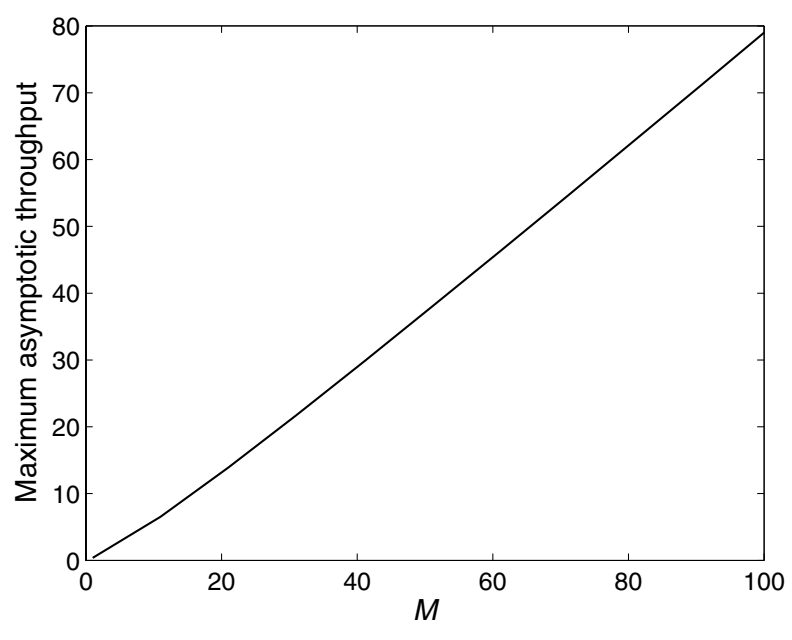

Fig. 8 Maximum asymptotic throughput by tuning $r$ versus $M$.

\section{Multipacket Reception in WLAN}

In this section, we analyze the performance of an MAC protocol for MPR with carrier-sensing. The reason why we are interested in this protocol is that this MPR MAC protocol closely follows the 802.11 DCF which employs the BEB as the backoff algorithm. Details of the MPR MAC protocol and the PHY implementation can be found in [5].

\subsection{Protocol Description}

The proposed protocol follows the 802.11 DCF RTS/CTS (Request To Send/Clear To Send) access mechanism closely, with extension to support MPR. We briefly describe the protocol in this subsection. For simplicity, we consider a single isolated $\mathrm{BSS}$ with an $\mathrm{AP}$ and $N$ associated client stations. We assume that the AP is the only station in the BSS with the capability to receive up to $M(M \geq 1)$ packets simultaneously.

Figure 9 illustrates the protocol operation. A station with a packet to transmit first sends an RTS frame to the AP. In our MPR MAC model, when multiple stations transmit RTS frames at the same time, the AP can successfully detect all the RTS frames if and only if the number of RTSs is no more than $M$. When the number of transmitting stations exceeds $M$, collisions occur and the AP cannot decode any of the RTSs. The stations will retransmit their RTS frames after a backoff time period as the original 802.11 protocol. 


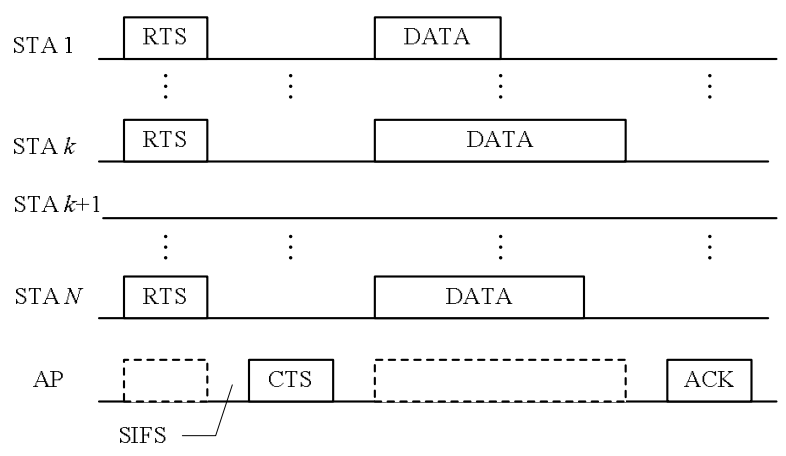

Fig. 9 Time line example for the MPR MAC.

When the AP detects the RTSs successfully, it responds, after a SIFS period, with a CTS frame that grants transmission permissions to all the requesting stations. Then the transmitting stations will start transmitting DATA frames after a SIFS, and the AP will acknowledge the reception of the DATA frames by an ACK frame.

\subsection{Performance Analysis}

In the following analysis, we assume the number of contending stations is fixed and that all stations operate in saturated conditions (i.e., they always have packets available for transmission). We also assume that all stations can hear each other (i.e., there is no hidden terminals) and the channel is perfect in that there is no packet loss due to fading.

We define "slot time" as the time interval between two consecutive backoff time counter decrements [13]. Note that the slot time we define here is not necessarily the constant slot time size . In $802.11 \mathrm{DCF}$, the backoff counter will be frozen when the channel is sensed busy. We define our slot time to include the frozen time and it is variable.

Although the MPR MAC is a carrier-sensing protocol, the analysis results we derive in Section 3 part 1 still apply. This is because the transmission probability, which is defined as the probability to transmit in an arbitrary slot, does not depend on the length of the slot. So (2) and (3) are true even in this carrier-sensing model.

We define the throughput $T$ to be the ratio of payload information bits being transmitted to the total amount of time spent to successfully transmit the payload. Therefore,

$$
\begin{aligned}
T & =\frac{E[\text { payload information bits transmitted in a slot time }]}{E[\text { length of a slot time }]} \\
& =\frac{\sum_{k=1}^{M} k P_{s k} P_{t r} E[L]}{\left(1-P_{t r}\right) \sigma+\sum_{k=1}^{M} P_{s k} P_{t r} T_{s k}+P_{t r}\left(1-P_{s}\right) T_{c}}
\end{aligned}
$$

where $P_{t r}$ and $P_{s k}$ are as defined in Section 3,E[L] is the average payload length in bits, $T_{s k}$ is the average slot time spent when there are successful $k$-packet transmissions, $T_{c}$ is the average slot time when there are collisions, and $P_{s}=\sum_{k=1}^{M} P_{s k}$ is the conditional probability of successful transmissions in a busy time slot. The general throughput expression (17) derived for MPR also incorporates the 802.11 case. In particular, when $M=1$, the MPR throughput reduces to the 802.11 throughput.

In our protocol, the RTS/CTS access scheme is employed. Therefore

$$
\left\{\begin{aligned}
T_{s k}= & R T S+S I F S+\delta+C T S+S I F S+\delta+H \\
& +E\left[L_{k}^{*}\right] / R+S I F S+\delta+A C K+D I F S+\delta \\
T_{c}= & R T S+D I F S+\delta
\end{aligned}\right.
$$

where $\delta$ is the propagation delay, $H=P H Y_{h d r}+M A C_{h d r}$ is the total overhead time to transmit the packet headers, $E\left[L_{k}^{*}\right]$ is the average length (in bits) of the longest payload involved in a $k$-packet simultaneous transmission, and $R$ is the data rate for payload transmission. In the following numerical investigations, we assume all packets have the same fixed length, i.e., the average length $E\left[L_{k}^{*}\right]=E[L]=L(1 \leq k \leq M)$ and $T_{s k}=T_{s}$, where $L$ and $T_{s}$ are constants.

To have a clear picture of the asymptotic behavior of the MPR MAC, again we resort to the infinite population model proposed in Section 3. We give the expression for the asymptotic throughput of the MPR MAC directly

$$
\lim _{N \rightarrow \infty} T=\frac{\sum_{k=1}^{M} k P_{s k} P_{t r} E[L]}{\left(1-P_{t r}\right) \sigma+\sum_{k=1}^{M} P_{s k} P_{t r} T_{s k}+P_{t r}\left(1-P_{s}\right) T_{c}}
$$

where all the symbols have already been defined previously in (17) except that $P_{t r}$ and $P_{s k}$ should be changed to

$$
P_{t r}=1-P\{X=0\}=1-e^{-\lambda}
$$

and

$$
P_{s k}=P\{X=k\} / P_{t r}=\frac{\lambda^{k}}{k !} e^{-\lambda} / P_{t r},
$$

respectively.

Parallel to the discussion in the non-carrier-sensing case, we plot the asymptotic throughput against $r$ for various $M$ in Fig. 10. The results shown here are from theoretical analysis and the parameter settings we used here are basically those from $802.11 \mathrm{~g}$ with some modifications as stated in [5] except that there is no retry limit here. From this figure, we can see that there still exists an optimal $r$ for each curve. The approach to achieve the best throughput performance by adjusting $r$ in MPR MAC is analogous to the one we mentioned in Section 3 . However, from the engineering's point of view, we argue that BEB (i.e., $r=2$ ) already achieves a close-to-optimal throughput for a large variety of $M$. Results show that about $45 \%$ increase in the maximum asymptotic throughput can be achieved when $M=2$ compared with $802.11 \mathrm{~g}$. Similar to the conclusions we made for the non-carrier-sensing system, Fig. 11 shows that the maximum asymptotic throughput increases roughly linearly with $M$. 


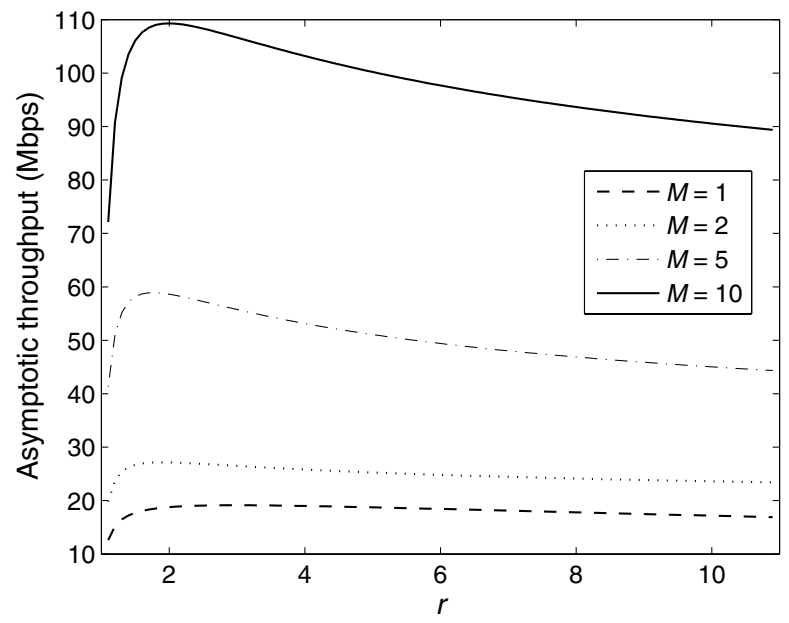

Fig. 10 Asymptotic throughput versus backoff factor $r$ for the MPR MAC.

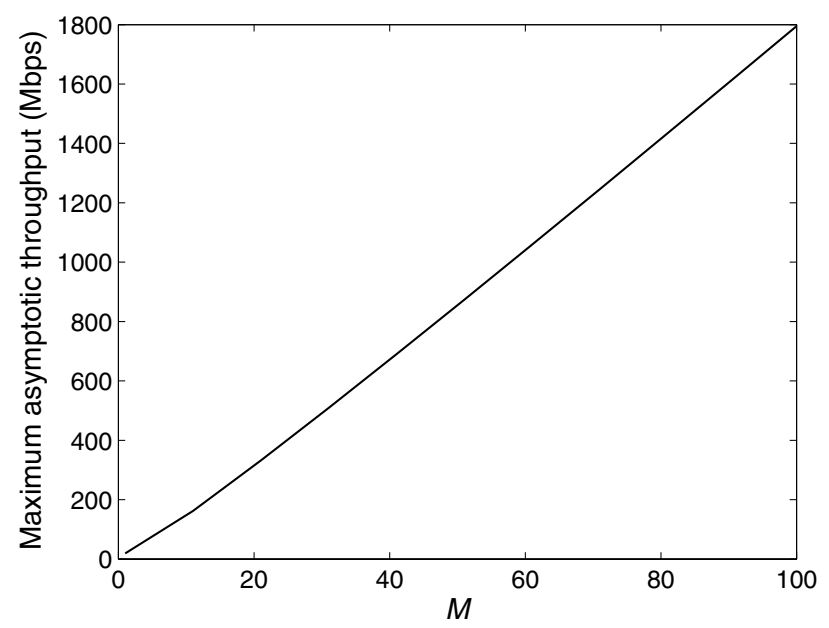

Fig. 11 Maximum asymptotic throughput versus $M$ for the MPR MAC (ignoring the address increase in CTS and ACK).

\section{Conclusion}

This paper has investigated the performance of EB in random-access networks with MPR capability that allows $M$ packets to be simultaneously transmitted without collision. Both carrier-sensing and non-carrier-sensing systems have been studied. Insights gained from our analysis are useful for system design in terms of setting the correct operating parameters. Extensive simulations have also validated the accuracy of our theoretical analysis.

We have derived the throughput expressions for both carrier-sensing and non-carrier-sensing systems under saturated-traffic condition, and have analyzed the asymptotic behavior of EB with MPR under infinite-population assumption. In both systems, the collision probability $p_{c}$ converges to $1 / r$ regardless of $M$ and the throughput given $M$ converges to a constant as $N$ increases (albeit different constants for the two systems). With the help of our throughput expression, we have also analyzed the variation of normalized throughput with respect to $M$ and the variation of throughput with respect to $r$ given $M$. Based on the infinite population model, we observed that the optimal $r$ increases with $M$ for non-carrier-sensing systems and BEB is close to optimal for carrier-sensing systems. We have presented numerical results showing the possible improvements with the MPR capability. Our results show that for carrier-sensing systems, throughput improvement of $45 \%$ can be achieved with our proposed MPR MAC protocol when $M=2$ compared with the conventional $802.11 \mathrm{~g}$ protocol. For both carrier-sensing and non-carrier-sensing systems, network throughput increases almost linearly with $M$ for the infiniteuser-population case.

\section{References}

[1] J. Goodman, A. G. Greenberg, N. Madras, and P. March, "Stability of binary exponential backoff," J. $A C M$, vol. 35, no. 3, pp. 579-602, Jul. 1988.

[2] L. Tong, Q. Zhao, and G. Mergen, "Multipacket reception in random access wireless networks: From signal processing to optimal medium access control," IEEE Commun. Mag., vol. 39, no. 11, pp. 108-112, Nov. 2001.

[3] P. B. Rapajic and D. K. Borah, "Adaptive MMSE maximum likelihood CDMA multiuser detection," IEEE J. Select. Areas Commun., vol. 17, no. 12, pp. 2110-2122, Dec. 1999.

[4] S. Talwar, M. Viberg, and A. Paulraj, "Blind separation of synchronous co-channel digital signals using an antenna array, Part I: Algorithms," IEEE Trans. Signal Processing, vol. 44, no. 5, pp. 1184-1197, May 1996.

[5] P. X. Zheng, Y. J. Zhang and S. C. Liew, "Multipacket reception in wireless local area networks," Proc. IEEE ICC'06, Istanbul, Turkey, June, 2006.

[6] S. Ghez, S. Verdú, and S. Schwartz, "Stability properties of slotted Aloha with multipacket reception capability," IEEE Trans. Automat. Contr., vol. 33, no. 7, pp. 640-649, Jul. 1988.

[7] Q. Zhao and L. Tong, "A multiqueue service room MAC protocol for wireless networks with multipacket reception," IEEE/ACM Trans. Networking, vol. 11, pp. 125-137, Feb. 2003.

[8] Q. Zhao and L. Tong, "A dynamic queue protocol for multiaccess wireless networks with multipacket reception," IEEE Trans. Wireless Comm., vol. 3, no. 6, pp. 2221-2231, Nov. 2004.

[9] D. S. Chan and T. Berger, "Performance and Cross-Layer Design of CSMA for Wireless Networks with Multipacket Reception," Proc. of Asilomar Conf. on Signals, Systems and Computers, vol. 2, pp. 1917-1921, Nov. 2004.

[10] ANSI/IEEE Std 802.3-1985, IEEE standards for local area networks: carrier sense multiple access with collision detection (CSMA/CD) access method and physical layer specifications, 1985.

[11] IEEE Std 802.11-1997, IEEE Std 802.11-1997 Information Technology- telecommunications And Information exchange Between Systems-Local And Metropolitan Area Networksspecific Requirements-part 11: Wireless Lan Medium Access Control (MAC) And Physical Layer (PHY) Specifications, Nov. 1997.

[12] B. J. Kwak; N. O. Song, and L. E. Miller, "Performance analysis of exponential backoff," IEEE/ACM Trans. Networking, vol. 13, no. 2, pp. 343-355, Apr. 2005.

[13] G. Bianchi, "Performance analysis of the IEEE 802.11 distributed coordination function," IEEE J. . Select. Areas Commun., vol. 18, no. 3, pp. 535-547, Mar. 2000. 